\section{Enzymic Conversion of L-Rhamnulose to L-Fuculose in Escherichia coli}

IN a previous paper ${ }^{1}$ indirect adaptation to $\mathrm{D}$-arabinose induced by $\mathrm{L}$-rhamnose in some $\mathrm{D}$-arabinose negative strains of Escherichia coli has been reported. These strains when grown on L-rhamnose, metabolize $\mathrm{D}$-arabinose and utilize it for growth. This effect is not due to selection of mutants but to enzymic adaptation : induction of $\mathrm{D}$-arabinose isomerase by L-rhamnose has been demonstrated.

This indirect induction can possibly occur through the enzymic conversion of rhamnulose (phosphate) to fuculose (phosphate). Fuculose is known in fact to be an inducer of the metabolic enzymes of $D$-arabin$o^{2}{ }^{2}$. Rhamnulose and rhamnulose-phosphate are normal products of rhamnose metabolism in $E$. coli ${ }^{3}$.

Huang and Miller, in their studies on lactaldehyde metabolism 4 , also put forward the hypothesis that fuculose was formed from rhamnulose.

We have identified fuculose phosphate among the sugar phosphates obtained by incubating rhamnose with an homogenate of $E$. coli (strain 30 ) cells in the presence of adenosine triphosphate.

$E$. coli (strain 30) was grown on a synthetic medium containing 0.3 per cent rhamnose, and the cells were collected during exponential growth. Homogenates obtained by grinding with alumina or by the Hughes press were diluted with $1 \cdot 1$ per cent potassium chloride.

For the preparation of the sugar phosphates, rhamnose was at first isomerized to rhamnulose under the following conditions: rhamnose 0.35 m.mole, homogenate (containing $15-17 \mathrm{mgm}$. protein per ml.) $8 \mathrm{ml}$., $0.2 M$ borate buffer $30 \mathrm{ml}$., cobaltous sulphate, $10^{-4} M$ (final concentration). The reaction was followed by the method of Dische and Borenfreund ${ }^{5}$ for the determination of rhamnulose until the equilibrium was reached. At this point $0.35 \mathrm{~m}$.mole of adenosine triphosphate (as the disodium salt), $5 \mathrm{ml}$. of homogenate and magnesium chloride so as to give a final concentration of $10^{-4} M$ were added.

After 2-hr. incubation the reaction was stopped with trichloroacetic acid. The precipitate was removed by filtration and the filtrate was brought to $p H$. Sugar phosphates were precipitated as the barium salts with ethanol and the precipitate was dissolved in $0.1 M$ acetic acid. Barium was precipitated as sulphate and the nucleotides adsorbed on charcoal until the absorption at $260 \mathrm{~m} \mu$ disappeared.

Sugar phosphates were chromatographed on What. man No. 1 paper with 80 per cent ethanol containing 0.8 per cent acetic acid. Two yellow and a green spot were obtained with the orcinol reagent ${ }^{6}$. The green spot was due to free rhamnulose; the others which were due to sugar phosphates also appeared with the ammonium molybdate reagent?

The sugar phosphates corresponding to the yellow spots were eluted from paper and hydrolysed with acid phosphatase ('Polidase $S$ ', Schwarz). The free sugars were chromatographed on Whatman No. 4 paper with benzene-ethanol-water 169: $47: 15 \mathrm{v} / \mathrm{v}$ using authentic samples of rhamnulose and fuculose as standards.

After hydrolysis, the slow-moving yellow spot gave with the orcinol reagent a yellow and a green spot: the first one had the same $R_{F}$ and colour as fuculose, the second as rhamnulose. The fast-moving yellow spot gave one green spot moving as rhamnulose.

The sugar moving as fuculose was eluted from the paper. By the cysteine carbazole reaction it gave a red colour with an absorption maximum at $550 \mathrm{~m} \mu$. By the orcinol-ferric chloride reaction ${ }^{8}$ it showed absorption maxima at 425 and $520 \mathrm{~m} \mu$ like all ketomethylpentoses. By the Dische and Shettles reaction ${ }^{9}$ it showed the $400 \mathrm{~m} \mu$ maximum characteristic of methylpentoses.

This sugar was also treated with an homogenate of $E$. coli 30 cells containing $D$-arabinose isomerase. After this treatment, two sugars could be detected by paper chromatography with butanol-acetic acidwater $4: 1: 5 \mathrm{v} / \mathrm{v}$ as solvent, using the benzidine spray reagent; these sugars showed the same chromatographic behaviour as fucose and fuculose respectively.

Fuculose phosphate, which we have tentatively identified among the sugar phosphates obtained from rhamnose, is probably formed by the inversion of carbon four of rhamnulose phosphate. \section{Laura Frontali
Giorgio Tecce}

National Institute of Nutrition,

Institute of General Physiology, University of Rome.

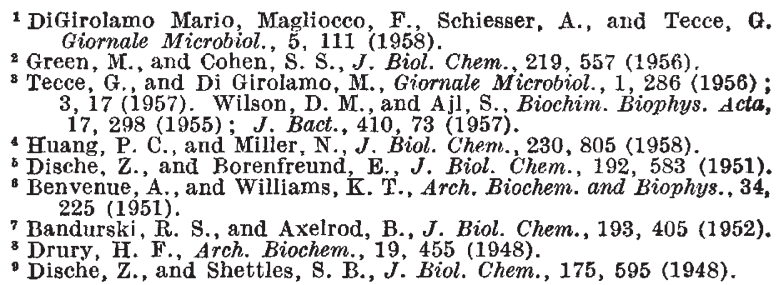

\section{Ionized Calcium in Biological Media}

THE unsatisfactory status of the important problem of the determination of ionized calcium in biological media has recently been re-appraised by $W$. F. and M. S. Neuman ${ }^{1}$. After critical examination of methods then existing and of available data on the medium most generally investigated, serum, they state that "the amount of ionized calcium in normal serum now seems to be pretty well established-approximately $1 \cdot 3 \mathrm{mM} /$ litre."

Using a direct general method, quite recently developed, which is based on the absorbance of metal (or $p M$ ) indicators at two wave-lengths ${ }^{2}$, we have redetermined the concentration of ionized calcium in the serum of normal adults using murexide as indicator. The effect of light scattering by serum, and of its variation with the wave-length, is eliminated in this two-wave-length method by the use of serum as a blank at each wave-length. Aniondye binding has also been considered by investigating solutions of Armour serum albumin and apparently is not of significance in this method.

Samples were taken at random from healthy student nurses. Equal volumes of the serums were pooled as a means of obtaining the equivalent of an average normal serum and were analysed almost immediately after being collected. This simple approach was used in order to make a comparison of the results obtained by the new method with those adopted by authorities in the field.

In the following table the values for the formation constants $K_{f}$ used for the calcium murexide complex correspond ${ }^{2}$ to ionic strength 0.15 and the measured $p \mathrm{H}$ value of each pooled serum (found to be within $0.2 p \mathrm{H}$ of the normal). 